\title{
Théologiques
}

\section{L'art et la Bible au risque de leurs interprètes}

Dialogue interdisciplinaire entre un exégète biblique et un

historien de l'art (Post-scriptum)

\section{Alain Gignac et Alain Laframboise}

Volume 17, numéro 2, 2009

Querelles d'images?

URI : https://id.erudit.org/iderudit/044067ar

DOI : https://doi.org/10.7202/044067ar

Aller au sommaire du numéro

Éditeur(s)

Faculté de théologie et de sciences des religions, Université de Montréal

ISSN

1188-7109 (imprimé)

1492-1413 (numérique)

Découvrir la revue

Citer cet article

Gignac, A. \& Laframboise, A. (2009). L'art et la Bible au risque de leurs interprètes : dialogue interdisciplinaire entre un exégète biblique et un historien de l'art (Post-scriptum). Théologiques, 17(2), 157-167.

https://doi.org/10.7202/044067ar 
Théologiques $17, \mathrm{n}^{\circ} 2$ (2009) p. 157-167

\title{
L'art et la Bible au risque de leurs interprètes
}

\section{Dialogue interdisciplinaire entre un exégète biblique et un historien de l'art (Post-scriptum $)^{1}$}

\author{
Alain GIGNAC* \\ Théologie et sciences des religions \\ Université de Montréal \\ Alain LAFRAMBOISE* \\ Histoire de l'art \\ Université de Montréal
}

1. Le présent essai, qui emprunte au genre littéraire "dialogue», fait suite aux deux articles d'Alain Gignac (études bibliques): «Caïn, protégé du Seigneur? Les voix de Gn 4,1-16 dans une perspective narratologique» et d'Alain Laframboise (histoire de l'art): «Images d'un meurtre. La Genèse des artistes». Ces deux articles ont été élaborés parallèlement; ils avaient pour "prétexte» l'histoire de Caïn et Abel et comme point commun une perspective narratologique. Après avoir pris connaissance du texte de l'autre, les deux auteurs ont voulu poursuivre la réflexion interdisciplinaire. Peuton identifier des passerelles entre les univers de la théologie et de l'histoire de l'art ? Des collaborations possibles? Qu'est-ce qui, dans le texte de l'un, a trouvé écho dans les préoccupations spécifiques de l'autre?

* Alain Gignac est professeur agrégé à la Faculté de théologie et de sciences des religions de l'Université de Montréal. Ses recherches portent sur la narratologie appliquée aux textes discursifs du Nouveau Testament (Lettres aux Galates et aux Romains), ainsi que sur les lectures que font des lettres de Paul les philosophes politiques contemporains (Badiou, Agamben, etc.). Il a récemment publié (2009) "La mise en scène de $\mathrm{Rm}$ 7,7-8,4. Une approche narrative et synchronique », dans U. Schnelle, dir., The Letter to the Romans. Colloquium Biblicum Lovaniense (Leuven, 25-27 juillet 2007), Leuven, Peeters (BETL; 226), p. 113-134.

* Alain Laframboise est professeur honoraire au Département d'histoire de l'art et d'études cinématographiques de l'Université de Montréal. Ses recherches ont surtout porté sur l'art italien de la Renaissance et du maniérisme. Il s'est beaucoup intéressé à la question de l'istoria, soit tout ce travail qui concerne le passage d'un récit du texte à l'image et qui a été, dès le début du Quattrocento, au cœur des réflexions tant des praticiens que des théoriciens de l'art. Il a notamment publié (1989) Istoria et théorie de l'art. Italie $\mathrm{XV}^{e}, \mathrm{XVI}^{e}$ siècles, Montréal, Presses de l'Université de Montréal. 
Gignac: La problématique du numéro de Théologiques me conduit à poser carrément la question de la collaboration interdisciplinaire: que peut attendre l'historien de l'art du bibliste? À l'inverse, il me semble que l'art ouvre au théologien le champ immense de la réception contextualisée du texte biblique, de ses multiples relectures à travers les siècles. Pour moi, l'artiste est un interprète, éventuellement conformiste ou au contraire provocateur et subversif, dont la théologie doit entendre la voix.

Laframboise: La réception des textes et celle des images nous permettent de constater, de part et d'autre, à quel point les sources textuelles ont été malléables pour les exégètes et les artistes. Les artistes ont pris les libertés que leur époque leur autorisait, c'est-à-dire en fonction de la lecture qu'elle faisait des textes sacrés, apocryphes et de tout ce qu'une tradition, savante ou populaire, les incitait à «inventer». Ils font, pour reprendre les mots d'Erwin Panofsky, mais sans adhérer à sa vision essentialiste, avec l'histoire des styles qui les incite à représenter les objets et les événements suivant des formes qui soient recevables par leurs contemporains, avec l'histoire des types qui a à voir avec la compréhension des thèmes et des concepts dans diverses conditions historiques et avec l'histoire des symptômes culturels qui s'exprime à travers des formes et des concepts spécifiques. L'historien de l'art a énormément à tirer d'un savoir qui lui révèle la sédimentation des exégèses textuelles. Il ne peut absolument pas fonctionner en circuit fermé. Il doit faire rhizome. Au-delà de l'approche panofskienne, il doit agir comme Aby Warburg ${ }^{2}$ et cultiver les relations associatives entre les savoirs, rechercher un monde ouvert de relations multiples sur le plus de champs possibles; sa perspective doit être davantage celle de l'anthropologue du visuel que celle de l'historien de l'art classique.

Gignac: Plusieurs notions développées en histoire de l'art semblent trouver leur correspondant dans la théorie littéraire qui est mon lieu propre (je travaille les textes). L'admoniteur m'intéresse particulièrement. Peut-on le comparer au narrateur, un personnage qu'on oublie trop souvent, à moins qu'il soit très intrusif ? L'admoniteur et le narrateur sont des figures (picturales ou textuelles) distanciées de l'auteur (peintre ou écrivain). Par ailleurs, dans le bas-relief de Ghiberti, pourrait-on considérer le personnage divin (au second plan, à droite), qui désigne à la fois le meurtre et possiblement l'exil, comme l'admoniteur qui donne la clé de lecture? En ce cas, l'artiste aurait choisi de nous raconter le récit du point de vue de Dieu...

2. Aby Warburg (1866-1929) est le véritable fondateur de la discipline iconologique dans un contexte germanique de Kulturwissenchaft. 
Laframboise: La figure que vous évoquez ici me semble concentrée sur le dernier épisode représenté, soit l'exil de Caïn. L'admoniteur, dans la perspective d'Alberti, s'adresse au spectateur pour diriger sa lecture. En ce sens, il peut difficilement, même s'il existe des exceptions, être à la fois acteur et commentateur. Plus encore un acteur principal. L'admoniteur regarde avec le spectateur. Puisque l'admoniteur est un dispositif mis en place par l'artiste, pour éclairer une mise en scène que ce dernier a composée, organisée, c'est d'abord le point de vue de l'artiste interprète du récit qu'il met en évidence.

Gignac: Sur ce thème de Caïn, $y$ aurait-il un exemple autre que ceux dont vous avez traités, où un personnage, "ficelle» ou "figurant ", indique le point de vue de l'auteur, la clé interprétative du tableau, la porte d'entrée dans l'œuvre picturale?

Laframboise: On touche toujours à des limites avec l'histoire de Caïn et Abel, le meurtre étant commis à l'écart, dans les champs. Le seul témoin possible, hormis le spectateur, paradoxalement présent, étant la divinité. Ce qui ne veut pas dire que, traitant d'autres thèmes, les artistes n'ont pas eu recours à des montages anachroniques, dont ils connaissaient bien les effets rhétoriques. Ils ont souvent mis en présence des personnages appartenant à des époques et à des récits différents afin d'orienter la lecture d'une image. Si les correspondances entre l'histoire de l'art et la théorie littéraire existent, certes, les équivalences apparaissent parfois difficiles à déterminer. Avec les «reprises figuratives ", comme vous le dites si justement, que l'on peut rapprocher des généalogies interprétatives, le programme iconographique et la diversité des solutions stylistiques organisent et réorganisent les articulations des sources textuelles. C'est alors que les parcours du regard viennent diriger l'interprétation. Mais j'insiste sur le fait que le spectateur a besoin de reconnaître le récit, soit les personnages, l'intrigue, le cheminement psychologique, pour comprendre ce qu'on lui montre. L'image prend appui sur une histoire.

Gignac: En relisant les deux articles, je prends mieux conscience du dilemme de l'artiste: là où le texte valorise la parole (monologue ou dialogue), avec finalement peu d'indications corporelles et de descriptions des mouvements, la représentation visuelle ne conserve que la gestuelle. Cela est très frappant, tant chez Ghiberti et Jacopo della Quercia que chez Titien.

Laframboise: La représentation visuelle «conserve» la gestuelle et ajoute le style (le traitement formel) au texte. En ce sens que l'artiste appartient à une école, à une tradition. Suivant les époques, il dispose de ressources variables. L'artiste byzantin n'aura pas les mêmes priorités et les 
mêmes objectifs que celui qui, à la Renaissance, se sert de la perspective et peut donner à ses figures du relief et un vaste registre d'expressions. Mais la gestuelle n'est jamais aussi transparente qu'on serait tenté de le croire. Michael Baxandall (1972) nous a montré que s'il n'y a pas de dictionnaire pour le langage des gestes à la Renaissance, des clés d'interprétation se trouvent, par exemple, dans les manuels rédigés à l'usage des prédicateurs, ou dans ceux indiquant les attitudes correctes recommandées aux jeunes filles, publiés au XVe siècle. Jean-Claude Schmitt (1990) nous a fait comprendre que, de l'Antiquité tardive jusqu'au Moyen Âge central, il n'existe pas de gestes «naturels» dans le monde des images, mais que ceux-ci sont à interpréter dans un contexte figuratif et culturel complexe. Sharon Fermor (1993), pour retenir une démonstration plus resserrée, a bien fait voir que les critères de féminité et de masculinité, de grâce et de virilité, reliés à la danse au XVI siècle (et plus généralement aux usages courtisans) étaient les mêmes que ceux qu'appliquaient des peintres comme le Titien ou Rosso dans la représentation des gestes et des mouvements de leurs figures féminines et masculines. Ainsi se glissent dans les images des éléments à connotation idéologique implicitement accessibles aux spectateurs contemporains de ces œuvres, mais qui demandent qu'on les redécouvre aujourd'hui.

Gignac: Un autre point qui me frappe: l'exégète et l'historien d'art doivent tenir compte, dans leur essai d'interprétation, de trois niveaux de complexité croissante: 1) l'œuvre elle-même, dans sa synchronicité, telle qu'elle se donne à lire ou à voir aujourd'hui, dans son dispositif littéraire ou visuel ; 2) son contexte historique, qui donne des clés d'interprétation et incarne l'œuvre dans un moment culturel; 3) l'histoire de l'interprétation, enfin, dans sa «diachronicité", qui permet de tracer une généalogie des influences, tant en amont qu'en aval.

Laframboise: Je suis absolument d'accord. Comment faire autrement? J'aurais envie d'insister sur la compréhension du contexte historique dans la mesure où, comme l'ont dit et répété de nombreux théoriciens, il existe un code d'utilisation symbolique de l'objet (texte, image) qui ne rend possible son déchiffrement que dans la mesure où le plan de connotation idéologique est entendu pleinement.

Gignac: Je fais allusion, dans mon texte, à une lecture monologique qui tend, dans l'histoire de l'interprétation, à réduire à une seule voix la clé d'interprétation d'un texte biblique - par contraste à une lecture dialogique, qui met en relief les multiples voix qui cherchent à s'y faire entendre. Existe-t-il, vis-à-vis d'un tableau ou d'un bas-relief, la même alternative? Est-ce une préoccupation pour les historiens de l'art? 
Laframboise: C'en est une. Toute image apparaît et s'insère dans un ensemble d'images. Plus trivialement, on parlera d'écoles, de traditions, de contextes qui influencent le travail des artistes. À la Renaissance, chaque artiste conçoit sa production comme s'inscrivant à la fois dans l'actualité artistique aussi bien que dans l'histoire des œuvres. Il existe donc des relations entre les œuvres, tant dans l'esprit des artistes que dans celui des amateurs d'art éclairés. En 1550, Vasari, le premier historien de l'art de l'ère moderne, proposera aux artistes de concevoir leurs créations comme une contribution à l'histoire du progrès artistique ${ }^{3}$. On a parlé des images savantes des artistes du XVI e siècle, de leur pratique de la citation, tantôt subtile, tantôt affichée, des grands maîtres tels Michel-Ange et Raphaël, ou parfois de leur subversion des lois établies des genres. Ce sont des pratiques qu'aujourd'hui on nomme "métaphores figuratives».

Gignac: Dans l'histoire de l'art, existe-t-il un artiste qui insiste plus sur le cheminement de Caïn qui advient à sa subjectivité, malgré et peut-être à cause de son meurtre fratricide - la lecture que je mets de l'avant dans mon article? On sait qu'en littérature, le poème de Hugo sur "La conscience » (de Caïn) propose une suite au récit biblique qui s'attarde sur la culpabilité du personnage. Ou encore, un artiste a-t-il considéré d'un point de vue positif Caïn, dans sa relation à Dieu qui le protège?

Laframboise: Il serait téméraire d'avancer qu'il n'en existe aucune. Cependant, il serait toutefois fort étonnant que les représentations artistiques de cet épisode biblique aient échappé à la lecture moralisante que vous identifiez comme déterminante au fil de l'histoire des exégèses. Mais voilà qui nous ramène au cœur de la question de l'interprétation des images. Certaines œuvres résistent à toutes les tentatives, si érudites soient-elles. C'est le cas de La Tempête de Giorgione, par exemple, dont la fortune critique est titanesque et les lectures savantes qui en sont faites des plus disparates. On l'a interprétée tantôt comme un sujet profane, tantôt comme une histoire sacrée. Plusieurs exégètes y voient Adam, Ève et leur premier fils, Caïn. Qui oserait affirmer qu'il existe une correspondance univoque entre une œuvre figurative et son «sujet»?

Gignac: L'idéal d'Alberti, «d'une peinture qui ne trahisse pas le textesource ", me rappelle un peu l'approche pas très lointaine en exégèse (et peut-être pas partout dépassée), profondément marquée par le positivisme,

3. Giorgio Vasari (1511-1574), Vite de' più eccellenti architetti, scultori e pittori (1550). Comme tous ses contemporains, Vasari adopte une vision évolutionniste de l'art, inspiré des auteurs romains de l'Antiquité. 
d'une explication et donc d'une interprétation unique d'un texte, parce qu'objective et scientifique. Or, toute interprétation donne vie à un texte mais est obligée, pour ce faire, d'aller plus loin, et donc de le trahir. Et c'est là l'intérêt de l'art pour le théologien: «l'artiste doit fatalement inventer ce que le texte ne lui livre pas » (mise en scène, composition, décor, gestuelle), choisir (le point de vue et la scène) et suppléer à ce qu'il ne peut rendre (la ou les voix, ainsi que le déroulement diachronique). Ce qui m'intéresse, justement, ce n'est pas la fidélité du peintre (n'en déplaise à Alberti), mais sa créativité et son acte de lecture. Quel point de vue adopte-t-il ?

Laframboise: L'artiste n'a pas le choix, il est obligé d'inventer. Il ne peut qu'inventer. Le De Pictura d'Alberti constitue véritablement le tout premier traité sur la peinture de l'ère moderne. Il n'y a rien d'étonnant à ce qu'il ait privilégié la question de la vérité du sujet dans la perspective où les œuvres devaient servir à enseigner aussi bien aux doctes qu'aux ignorants. De plus, il proposait une nouvelle définition de l'artiste, non plus seulement habile artisan mais artiste savant ayant accès à cette culture humaniste jusqu'alors réservée aux lettrés et aux membres des arts libéraux. Alberti mais aussi les trattatistes de la Contre-Réforme à sa suite ont écrit comme si tout ce passage du texte à l'image allait de soi, d'abord préoccupés par cette injonction: l'œuvre doit dire vrai afin d'instruire les spectateurs.

Gignac: Vous parlez des «parcours» possibles du regard sur la toile, permettant de réinstaurer une relative diachronie de l'acte de lecture du tableau. Pluralité de parcours, parmi lesquels on peut éventuellement distinguer un parcours principal suggéré par l'artiste, à partir d'une analyse critique de la composition. Il y a analogie avec le texte: le lecteur scientifique ou savant prétend reconstituer un parcours de lecture suggéré par l'œuvre, mais ne peut imposer sa reconstitution comme prescriptive. De toute manière, tant dans le texte que dans le dispositif visuel, l'auteur a pu mettre à son insu plus qu'il n'y pensait, ou encore, le lecteur peut résister au parcours suggéré par l'auteur et "essayer» des parcours alternatifs. Tant en art qu'en exégèse, cette question des points de vue à identifier devient cruciale.

Par ailleurs, cette question des parcours fait surgir une interrogation: la sémiotique (de Greimas) ne serait-elle pas un meilleur outil méthodologique pour qu'exégèse et histoire de l'art entrent en dialogue?

Laframboise: Elle leur procurerait un langage commun, mais sans doute au prix d'une réduction ou d'une reddition de la spécificité des approches. Réaliserait-on l'ambition universelle du projet structuraliste 
greimassien? Parviendrait-on à atteindre cette objectivité scientifique des analyses rêvée par le théoricien?

Gignac: J'enregistre votre réticence par rapport au projet de Greimas, projet sans doute un peu totalitaire, avec le recul, et qui évacuait trop radicalement l'historicité. Mais puis-je me faire l'avocat de la défense? Dans votre article, vous soulevez la question de la nécessité, pour comprendre l'œuvre d'art, de connaître les prêches de l'époque. Plus haut dans notre entretien, vous avez insisté sur la compréhension du contexte historique. Or, le détour par la médiation historique, forcément érudit, s'il est important, est-il nécessaire? Le spectateur n'est-il pas outillé par l'image elle-même pour voir, de manière immanente?

Laframboise: Pas plus que ne l'est le lecteur de la Bible par le seul texte. Quelle merveille ce serait si nous accédions ainsi aux textes et aux œuvres par la seule grâce de l'immanence! Hormis la conviction de certains sémiologues dévoyés qui semblent croire que l'œuvre leur livrera tout au premier coup d'œil, je ne crois pas que l'on aille, par pareille voie, au-delà d'une analyse anecdotique et formelle limitée. L'analyse sémiotique, comme la définissait Louis Marin dans ses Études sémiologiques (1971), implique que l'objet porte en lui-même les traces de sa création dans une conjoncture socio-historique particulière. La sémiotique lui apparaissait, par nécessité de méthode, théorie critique des idéologies. Votre article est la démonstration même de la multiplicité des interprétations du mythe de Caïn et Abel. Il montre comment ces diverses exégèses en éclairent et nourrissent aujourd'hui notre compréhension.

Gignac: La difficulté, en ce qui concerne la Bible hébraïque, est de dater la rédaction des textes - la question se pose différemment pour le Nouveau Testament, dont l'ancrage socio-historique est bien situé et documenté, au Ir siècle. Il est d'autant plus difficile de dater les textes qu'en arrière-plan, c'est toute l'historiographie biblique et l'historicité de ses «événements» qui sont actuellement remises en question par les spécialistes (Finkelstein et Silberman 2004; Abadie 2009). Pour Caïn et Abel, à quelle couche rédactionnelle se rattache l'épisode? Quelle époque historique est susceptible d'éclairer notre compréhension du mythe: la cour salomonienne (en autant qu'elle ait existé, au $\mathrm{X}^{\mathrm{e}}$ siècle), la réforme deutéronomiste ( $\mathrm{VII}^{\mathrm{e}}$ siècle), le retour d'exil ( $\mathrm{V}^{\mathrm{e}}$ siècle) ? Comment établir le programme idéologique du récit en fonction de son milieu de vie? Le texte de Gn 4 suppose une codification des sacrifices, ainsi que l'existence d'un corpus législatif mais cela laisse encore un large spectre temporel. Par ailleurs, à travers la 
trajectoire qui va des diverses versions orales à sa mise par écrit définitive, il est clair qu'un même texte a pu être porté et remanié en fonction de visées diverses (à moins d'homogénéiser de manière outrancière l'histoire du Proche-Orient ancien).

Laframboise: "Comment établir le programme idéologique du récit... » Il en va de même pour l'historien de l'art. Vous soulignez là les risques du travail de l'exégète. Si grande soit son ambition, il lui faut choisir comment mener son investigation: comment fonder son herméneutique et révéler les réseaux symboliques qu'elle implique, comment opérer la "reconstruction » de phénomènes historiques, comment équilibrer enquête historique et analyse formelle, quelles limites fixer aux domaines chronologique et spatial, quelle part accorder aux récurrences typologiques par rapport aux connaissances historiques, etc.

L'exégète biblique trouve sans doute sa "liberté herméneutique ", d'une part, dans sa recherche pour se rapprocher des textes-sources - ainsi la prédominance accordée au texte massorétique - et, d'autre part, dans l'étude historique des interprétations. L'histoire de l'art s'intéresse de plus en plus à ce qu'on a appelé les circuits des images, d'abord le grand circuit public des masses longtemps composées d'illettrés (églises, palais publics, tavernes) et celui réservé à une élite cultivée et fortunée (les grandes résidences privées des princes et des prélats). Ces deux groupes de destinataires impliquent des codes culturels des plus disparates. Protégé de Laurent de Médicis, le chanoine Marsile Ficin, qui enseignait la pensée néoplatonicienne aux humanistes florentins, n'entendait pas l'histoire de Caïn et d'Abel de la même manière que le prêcheur s'adressant aux fidèles rassemblés dans son église. Jusqu'à quel point ces deux circuits, populaire et privé, se manifestent-ils, quand on envisage les récits bibliques?

Gignac: Vous ouvrez là un vaste chantier pour les exégètes. Dans notre analyse minutieuse des textes, tant du point de vue historique que littéraire, assumons-nous suffisamment les interrogations portées par les croyants, pour qui ces textes servent de guide dans leur itinéraire spirituel, ou par les agnostiques, pour qui ces mêmes textes demeurent des classiques susceptibles d'enrichir leur quête de sens? Bien plus, comment prenons-nous en compte les pratiques de lecture populaires de la Bible? Le mouvement latino-américain de la théologie de la libération s'est construit à partir des relectures des récits bibliques par les communautés de base, en fonction des préoccupations concrètes des gens et des défis économico-politiques qu'ils rencontraient (Segundo 1988). Il me semble que l'universitaire ne 
doit pas tant lire les textes pour les gens, qu'avec eux. Or, la production de savoir biblique tend à fonctionner dans le circuit fermé universitaire, entre congrès spécialisés et revues savantes. Si la recherche biblique moderne peut être caractérisée comme le combat pour une lecture scientifique de la Bible affranchie du contrôle dogmatique de la théologie et des responsables ecclésiaux, il faut maintenant remettre en question le monopole universitaire de l'interprétation des textes, qui est devenu, lui aussi, «magistériel» et «dogmatique». Il y a là une question de pouvoir: qui valide l'interprétation des textes? D'un point de vue narratif, il est bien que je m'interroge sur le lecteur virtuel construit par la narration et sur ses éventuelles réactions; mais il conviendrait aussi que je vérifie comment les réels lecteurs du récit de Caïn et Abel, ou de Caïn et Dieu, selon qu'on voudra l'intituler, réagissent à la mise en intrigue. De fait, les universitaires ne sont pas si enfermés dans leur tour d'ivoire. Par exemple, les interprétations de la Bible sont actuellement traversées par le questionnement du féminisme, des cultural studies ou de la perspective post-coloniale. De même, Daniel Patte, qui pilote un ambitieux programme de relecture de la lettre aux Romains «à travers l'histoire et la culture », a forgé l'expression «Scriptural Criticism» pour désigner une interprétation biblique qui, sans brader la rigueur scientifique, prend en compte l'expérience croyante et la vision du monde qui la structure (Grenholm et Patte 2000), tout en étant attentif à l'impact des hypothèses de lecture mises de l'avant.

Laframboise: On peut avoir l'impression que différemment des productions figuratives, lorsqu'il est question de périodes relativement courtes, les exégèses portant sur des textes sacrés s'organisent, provisoirement, à partir d'un même corpus textuel (qu'il provienne d'une ou de plusieurs sources). Alors qu'à moins qu'elle ne s'intéresse aux traités artistiques, l'histoire de l'art, quelle que soit la période qu'elle étudie, travaille sur des pièces singulières, chaque tableau, chaque sculpture fonctionnant selon ses propres règles sans livrer avec certitude aucune référence textuelle. A-t-on raison?

Gignac: La réponse dépend de la définition que l'on donne à "corpus textuel». Tout comme les historiens de l'art, nous pouvons examiner un texte en lui-même, en son fonctionnement singulier, ou encore le situer dans une séquence (narrative, poétique, discursive), dans une thématique ou dans son genre littéraire. Il existe donc plusieurs manières de déterminer un corpus. Pour reprendre le cas de Gn 4, j'ai examiné le récit dans mon article comme une pièce singulière "fonctionnant selon ses propres règles sans 
livrer avec certitude aucune référence » extra-textuelle. Situé dans une séquence, Gn 4 fait partie de la saga des origines (Gn 1-11); situé dans une thématique, Gn 4 pourrait être rapproché des textes bibliques qui réfléchissent sur la violence; du point de vue du genre littéraire, on pourrait le comparer à d'autres récits étiologiques. Pour reprendre un autre exemple tiré de mon champ de recherche, un texte de Paul comme l'hymne à l'amour (1Co 13) peut être appréhendé en lui-même; en sa séquence rhétorique immédiate (1Co 12-14) ou lointaine (1Co); dans l'ensemble de la production de l'auteur (les sept lettres dites authentiques: $\mathrm{Rm}, 1-2 \mathrm{Co}, \mathrm{Ga}, \mathrm{Ph}$, $1 \mathrm{Th}, \mathrm{Phm})$ - ce qui peut impliquer une éventuelle évolution de la pensée; enfin, par rapport aux hymnes du Nouveau Testament. La tendance actuelle de la recherche est d'expliquer un passage d'une lettre en faisant référence uniquement à cette lettre, ou du moins à la correspondance entre Paul et une communauté donnée, puisque la lettre est acte de communication qui correspond à une situation concrète.

Laframboise: Je crois que notre échange indique bien la proximité de nos objectifs et de nos approches.

Gignac: J'émets le souhait que la collaboration puisse s'intensifier entre historiens de l'art et commentateurs des textes bibliques. Dans cette ligne, je salue la collection "Triptyque ", chez Desclée de Brouwer, qui met en dialogue trois auteurs autour d'une œuvre - par exemple, un philosophe, un exégète et un historien de l'art ${ }^{4}$. Pour ma part, il me semble qu'il y a là un champ de recherche et d'enseignement, du côté de la théologie. Nos propres étudiants en théologie et en sciences des religions, souvent centrés, à juste titre, sur des questions actuelles, auraient intérêt à renouer avec leur héritage culturel chrétien.

Laframboise: De la même façon, l'historien de l'art en formation se doit d'acquérir, sans qu'elle soit aucunement exclusive, la connaissance de ce qu'on appelle, faute d'une meilleure expression, «la culture humaniste». Idéalement, ce savoir se doit d'être toujours en développement au même titre que l'acquisition d'une pensée critique portant sur les fins et les moyens de la discipline. On ne peut pas faire d'histoire de l'art sans connaître la Bible, les grands textes mythologiques et les œuvres littéraires qui ont marqué les grandes civilisations, pas plus qu'en ignorant l'actualité, toujours mouvante, des approches critiques.

4. La collection comprend huit titres mais semble malheureusement avoir été interrompue - voir les quelques exemples en bibliographie. 


\section{Références}

Abadie, P. (2009), L'Histoire d'Israël entre mémoire et relecture, Paris, Cerf (Lectio Divina; 229).

Alphant, M., G. LAFON et D. Arasse (2001), L'apparition à Marie-Madeleine - noli me tangere, Paris, Desclée de Brouwer (Triptyque).

BAXANDall, M. (1972), Painting and Experience in Fifteenth-Century Italy, Londres, Oxford University Press.

FERMOR, S. (1993), «Movement and Gender in Sixteenth-Century Italian Painting ", The Body Imaged, The Human Body Form and Visual Culture Since the Renaissance, Cambridge, Cambridge University Press, p. $129-145$.

Finkelstein, I. et N. A. Silberman (2004) [2002; anglais 2001], La Bible dévoilée. Les nouvelles révélations de l'archéologie, Paris, Gallimard (Folio Histoire; 127).

Grenholm, C. et D. Patte (2000), «Receptions, Critical Interpretations, and Scriptural Criticism ", dans C. GRENHOlm et D. PATTE, dir., Reading Israel in Romans. Legitimacy and Plausibility of Divergent Interpretations, Valley Forge, Trinity Press (Romans Through History and Cultures Series), p. 1-54.

Kéchichian, P., S. Breton et P. Morel (2001), La conversion de Paul, Paris, Desclée de Brouwer (Triptyque).

Klépront, C., M. Buhot de Launay et L. Weigert (2003), Judith et Holopherne, Paris, Desclée de Brouwer (Triptyque).

MARIN, L. (1971), Études sémiologiques, Paris, Klincksieck.

SCHEFER, J.-L., H.-D. SAFFrey et J.-C. LEBENSZTEjN (2001), La création d'Ève, Paris, Desclée de Brouwer (Triptyque).

Schмiтt, J.-C. (1990), La raison des gestes dans l'Occident médiéval, Paris, Gallimard.

Segundo, J. L. (1988), Le christianisme de Paul: l'histoire retrouvée, Paris, Cerf (Cogitatio Fidei; 151). 